\title{
Molecules
}

\section{Functionalized Folic Acid-Chitosan Nanoparticles for Encapsulation of Dietary Polyphenols}

\author{
P. Chanphai and H. A. Tajmir-Riahi*
}

Department of Chemistry-Biochemistry-Physics, Université du Québec à Trois-Rivières, C. P. 500, Trois-Rivières (Québec), Canada G9A 5H7

$\underline{\text { Key words: }}$ polyphenols; folic acid-chitosan; encapsulation; loading efficacy; TEM

Abbreviations: $\mathrm{Ch}$, chitosan, FA, folic acid; res, resveratrol; gen, genistein; cur, crucumin; LE, loading efficacy; FTIR, Fourier transform infrared; TEM, Transmission electron microscopy

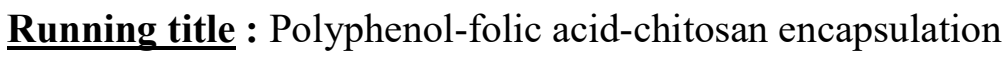

* Corresponding author: H. A. Tajmir-Riahi; Fax: 819-376-5084; Tel: 819-376-5011 (ext. 3310), e.mail: tajmirri@uqtr.ca 


\begin{abstract}
Encapsulation of antioxidant polyphenols leads to increase solubility and bioavailability of these micronutrients in solution. The encapsulation of antioxidant resveratrol, genistein and curcumin by folic acid-chitosan nanocapsules was studied in aqueous solution, using multiple spectroscopic methods, TEM images. Structural analysis showed that polyphenol bindings are via hydrophilic, hydrophobic and $\mathrm{H}$-bonding contacts with resveratrol forming more stable conjugates. As chitosan size increased, the binding efficacy and stability of polyphenol-polymer adducts were increased. Polyphenol binding induced major alterations of chitosan morphology. Chitosan nanoparticles are capable of delivery of polyphenols in vitro.
\end{abstract}




\section{Introduction}

Despite the health benefits associated with polyphenols, the bioavailability of many polyphenol limits their effects $[1,2]$. Problems with poor solubility fastmetabolism and food preparation techniques limit the bioavailability and bioactivity of these dietary micronutrients [3]. Encapsulation of polyphenols has shown to protect and increase bioavailability of these dietary compounds and to enhance their anticancer activity [4-6].

Folic acid-conjugated with biodegradable chitosan nanoparticles were often used as drug delivery tools [7-14]. Antioxidant polyphenols, resveratrol, genistein and curcumin (Scheme 1) shown major protection against cancer, cardiovascular diseases and DNA damage $[15,16]$. However, due to insolubility of these polyphenols, the bioavailability of these micronutrients is limited, while encapsulation can increase their solubility and enhances bioavailability in solution [1,2]. Folic acid-chitosan conjugates have the desired properties for safe use as pharmaceutical drug delivery tools. They show great utility in controlled release and targeting studies of almost all class of bioactive molecules [7-14]. Thus, it was of our interest to study the encapsulation of resveratrol, genistein and curcumin with folic acid-chitosan nanoconjugates in order to examine the efficacy of folic acid-chitosan nanocarriers in transporting polyphenols.

The encapsulation of resveratrol, genistein and curcumin with folic acid-chitosan nanocapsules is reported, using multiple spectroscopic methods, TEM analysis and molecular modeling. The loading efficacy and the effect of polyphenol encapsulation on polymer morphology is discussed here. 


\section{Experimental}

\subsection{Materials}

Highly purified resveratrol, genistein, curcumin and folic acid were purchased from Sigma Chemical Company (St-Louis, MO) and used as supplied. Purified chitosan 15 and $100 \mathrm{KDa}(90 \%$ deacetylation) were from Polysciences Inc. (Warrington, USA) and used as supplied. Other chemicals were of reagent grade and used without further purification.

\subsection{Preparation of Polyphenol-Acid-Chitosan Conjugates}

Preparation of folic acid-chitosan nanoconjugates were carried out according to the published method [17]. Polyphenol solution $(1 \mathrm{mM})$ was first prepared in Tris$\mathrm{HCl} /$ ethanol $50 \%$ and then diluted by serial dilution to different concentrations in Tris$\mathrm{HCl} /$ ethanol. Polyphenol-folic acid-chitosan conjugates were prepared by addition of polyphenol solution to folic-acid-chitosan nanocapsule in $10 \mathrm{mM}$ Tris- $\mathrm{HCl}$ at $\mathrm{pH} 7.2$ with occasional stirring to ensure the formation of a homogeneous solution.

\subsection{Fluorescence Spectroscopy}

Fluorimetric experiments were carried out on a Perkin-Elmer LS55 Spectrometer. Stock solutions of polyphenol $1 \mathrm{mM}$ were prepared at room temperature $\left(24 \pm 1^{\circ} \mathrm{C}\right)$. Various solutions of polyphenol $(100 \mu \mathrm{M})$ were prepared from the above stock solutions at $24 \pm 1{ }^{\circ} \mathrm{C}$. Solutions of folic acid-chitosan conjugate $(10$ to $200 \mu \mathrm{M})$ were prepared in $2 \%$ acetic acid and diluted in Tris- $\mathrm{HCl}(\mathrm{pH} .7 .2)$ at $24 \pm 1{ }^{\circ} \mathrm{C}$. Samples containing $0.4 \mathrm{ml}$ of the above polyphenol solution and various folic acid-chitosan solutions (10 to $200 \mu \mathrm{M}$ ) were mixed to obtain final polyphenol concentration of 30 $\mu \mathrm{M}$. The fluorescence spectra were recorded at $\lambda \mathrm{em}=420 \mathrm{~nm}$ (resveratrol), $375 \mathrm{~nm}$ 
(genistein ) and $365 \mathrm{~nm}$ (curcumin). The intensity of these bands were used to calculate the binding constant $(\mathrm{K})$ [18-22].

\subsection{FTIR Spectroscopic Measurements}

Infrared spectra were recorded on a FTIR spectrometer (Impact 420 model), equipped with deuterated triglycine sulphate (DTGS) detector and $\mathrm{KBr}$ beam splitter, using $\mathrm{AgBr}$ windows. Interferograms were accumulated over the spectral range 4000-600 $\mathrm{cm}^{-1}$ with a nominal resolution of $2 \mathrm{~cm}^{-1}$ and 100 scans. The difference spectra [(folic acid-chitosan solution + polyphenol solution $)-($ folic acid-chitosan solution)] were generated as reported [17].

\subsection{Transmission Electron Microscopy}

The TEM images were taken using a Philips EM 208S microscope operating at 180 $\mathrm{kV}$. The morphology of the polyphenol with folic acid-chitosan conjugates was monitored in aqueous solution at $\mathrm{pH} 7.2$, using transmission electron microscopy. One drop $(5-10 \mu \mathrm{L})$ of the freshly-prepared mixture [polyphenol solution $(60 \mu \mathrm{M})+$ folic acid- chitosan solution $(60 \mu \mathrm{M})]$ in Tris- $\mathrm{HCl}$ buffer $\left(24 \pm 1{ }^{\circ} \mathrm{C}\right)$ was deposited onto a glow-discharged carbon-coated electron microscopy grid. The excess liquid was absorbed by a piece of filter paper, and a drop of $2 \%$ uranyl acetate negative stain was added before drying at room temperature.

\subsection{Docking Study}

The docking studies were performed with ArgusLab 4.0.1 software (Mark A. Thompson, Planaria Software LLC, Seattle, Wa, http://www.arguslab.com). The chitosan and folic acid structures were obtained from literature report $[23,24]$ and the polyphenol three dimensional structures were generated from PM3 semi-empirical calculations using 
Chem3D Ultra 6.0. The docking runs were performed on the ArgusDock docking engine using regular precision with a maximum of 1000 candidate poses. The conformations were ranked using the Ascore scoring function, which estimates the free binding energy [24].

\section{Results and Discussion}

\subsection{TEM Analysis and Polyphenol-Folic Acid-Chitosan Morphology}

Evidence for polyphenol encapsulation by folic acid-chitosan nanoconjugates comes from transmission electron microscopic analysis. The shapes of free chitosan and folic acid-chitosan conjugates with their polyphenol complexes are shown in the TEM images (Fig. 1). TEM micrographs show that free chitosan and folic acid-chitosan conjugates show markedly different shape depending on its spherical-shaped size (Fig. $1 A$ and B) with smooth surface and narrow size distribution of about $90 \mathrm{~nm}[25,26]$. However, marked differences were observed in the morphology of the nanoconjugate aggregates. TEM images clearly showed the appearance of the aggregates of irregular shapes dispersed in solution when conjugated with polyphenols (Fig. 1C-E). In addition, the bound folic acid-chitosan with polyphenol showed major changes of the polymer morphological shape (Fig. 1A-E). An increase of the spherical-shaped aggregates can be seen from TEM micrograph, suggesting that the spherical shapes were lost in favor of less spherical-shaped in the polyphenol-folic acid-chitosan aggregates (Fig. 1B-E).The loss of the spherical shape of chitosan nanoparticles after complex formation with polyphenol is related to the polyphenol encapsulation. This is consistent with major particle size increase as encapsulation occurs (Fig. 1B-E). Polyphenol binding to folic acid- chitosan, which is a linear polysaccharide with multiple sites of interaction should 
be regarded as core-shell system with polyphenol (core) and acid-chitosan (shell) [2730]. The results suggest that the binding of polyphenol to folic acid-chitosan can play a role in altering the shape of the nanoparticles due to polyphenol encapsulation.

\subsection{Fluorescence Spectra and Binding Parameters of Polyphenol-Folic Acid-Chitosan} Nanocapsules

The titrations of polyphenols were done against various folic acid-chitosan concentrations, using polyphenols emission bands at 425 (resveratrol), $375 \mathrm{~nm}$ (genistein) and $365 \mathrm{~nm}$ (curcumin) [18-21]. When acid-chitosan interacts with polyphenol, fluorescence change depending on the impact of such interaction on the polymer conformation, or via direct quenching effect [22]. The decrease of fluorescence intensity of polyphenols has been monitored at $365-425 \mathrm{~nm}$ for polyphenol-acid-chitosan conjugates (Figs 2 and 3A-C). The plot of $F_{0} /\left(F_{0}-F\right)$ vs $1 /$ [acid-chitosan] is shown in Fig 2 and $3 A^{\prime}-C^{\prime}$. Assuming that the observed changes in fluorescence come from the interaction between the polyphenols and polymer conjugate, the quenching constant can be taken as the binding constant of the complex formation. The $K$ value obtained is the averages of four and six-replicate run for polyphenol-polymer systems (Table 1). The overall binding constants showing resveratrol forms more stable polymer conjugates than genistein and curcumin (Table 1). This indicates that polyphenol-polymer conjugate interactions involve both hydrophilic and hydrophobic contacts. The number of polyphenol molecules bound per polymer conjugate $(n)$ is calculated from $\log \left[\left(F_{0}-F\right) / F\right]$ $=\log K_{\mathrm{S}}+n \log$ [polyphenol] for the static quenching [22]. The $n$ values from the slope of the straight line plot showed 1.5 to 1 for polyphenol molecules that are bound per polymer molecule (Table 1). The stability of polyphenol-polymer was increased as the 
size of folic acid-chitosan conjugate increased (Table 1). In should be noted that a comparison between polyphenol-chitosan adducts and polyphenol-folic acid-chitosan conjugates showed larger loading efficacy and more stable polyphenol-polymer conjugates than polyphenol-chitosan complexes [31].

The loading efficacy for polyphenol-polymer conjugates was determined as reported [32]. The loading efficacy was estimated $65-50 \%$ for these polyphenol-chitosan conjugates. The loading efficacy enhanced as chitosan size of nanocapsule was increased (Table 1).

\subsection{Binding Analysis of Polyphenol-Folic Acid-Chitosan Nanoconjugates by FTIR}

\section{Spectroscopy}

The major spectral shifting and intensity variations of polymer amide I band at 1633-1620 $\mathrm{cm}^{-1}$ (mainly $\mathrm{C}=\mathrm{O}$ stretch) and amide II band at $1540-1520 \mathrm{~cm}^{-1}(\mathrm{C}-\mathrm{N}$ stretching coupled with $\mathrm{N}-\mathrm{H}$ bending modes) $[17,33]$ were used to characterize the nature of polyphenol-polymer interactions (Figures 4 and 5).

At low polyphenol concentration $(15 \mu \mathrm{M})$, a minor increase in the intensity was observed for the acid-chitosan amide I at $1633-1632 \mathrm{~cm}^{-1}$ and amide II at $1542-1540 \mathrm{~cm}^{-}$ 1, in the difference spectra of the polyphenols-polymer complexes (Figs 4 and 5, diff., 15 $\mu \mathbf{M})$. However, at higher polyphenol content $(60 \mu \mathrm{M})$, major decrease of intensity was observed for polymer amide I and amide II bands (Figs 4 and 5, diff. $60 \mu M$ ). The intensity variations were enhanced in the case of acid-chitosan-100, upon polyphenol conjugation (compare Figs 4 and 5 difference spectra). These negative features are related to the loss of intensity of the acid-chitosan vibrational frequencies upon polyphenol conjugation. The loss of intensity of the acid-chitosan amide I and amide II 
bands is due to polyphenol bindings to polymer $\mathrm{C}=\mathrm{O}, \mathrm{C}-\mathrm{N}$ and $\mathrm{N}-\mathrm{H}$ groups (hydrophilic interaction). The hydrophobic contacts are also observed between polyphenols and folic acid of polymer conjugates due to alterations of acid vibrational frequencies (Figs 4 and 5).

\subsection{Docking Study and the Bindings of Polyphenol to Folic Acid and Chitosan}

Since we could not locate the polyphenol binding sites on folic acid-chitosan conjugates, the locations of polyphenols on both folic acid and chitosan were compared here (Fig. 6). The docking results showed that polyphenols are weakly bonded to folic acid (Fig. 6A) and chitosan (Fig. 6 B). Polyphenols are surrounded by hydrophobic parts of folic acid with the free binding energy of -4.0 to $-3.30 \mathrm{kcal} / \mathrm{mol}$ (Fig. 6A). On the other hand, polyphenols are located on the surface of chitosan with the free binding energy of -4.1 to $-3.40 \mathrm{kcal} / \mathrm{mol}$ (Fig. 6B). Docking results show genistein binds folic acid and chitosan stronger than resveratrol and curcumin (Fig. 6). This is in contrast with the spectroscopic results that showed resveratrol forms more stable conjugate than genistein and curcumin (Table 1).

\section{Concluding Remarks}

Encapsulation of polyphenols enhances the bioavailability of these micronutrients in solution $[1-5,34]$. Folic acid functionalized drug delivery system based on resveratrol is known [35]. The binding analysis of resveratrol, genistein and curcumin with folic acidchitosan nanocapsules show that polyphenol-polymer conjugation is via hydrophilic, hydrophobic and H-bonding contacts with resveratrol forming stronger polymerconjugates. The stability and loading efficacy of polyphenol-polymer conjugates increased as folic acid-chitosan size was increased. Polyphenol encapsulation alters 
polymer morphology with an increase in the size of folic acid-chitosan aggregate due to polyphenol conjugation. A comparison between binding efficacy of polyphenol-chitosan adducts and polyphenol-folic acid-chitosan nanoconjugates shows larger stability and loading efficacy for polyphenols with folic acid-chitosan nanocapsules. Folic acidchitosan nanocarriers can deliver polyphenols and enhance the bioavailability of these dietary micronutrients.

\section{Acknowledgments}

The financial support of the Natural Sciences and Engineering Research Council of Canada (NSERC) is highly appreciated .

\section{References}

1. Bohn, T. Dietary factors affecting polyphenol bioavailability. Nutrition Rev. 2014, $72,429-452$.

2. D'Archivio, M,; Filesi, C.; Varì, R.; Scazzocchio, B.; Masella, R.

Bioavailability of the polyphenols: Status and controversies. Int. J. Mol. Sci.

2010, 11, 1321-1342.

3. Lu, W.; Kelly, A.L.; Miao, S. Emulsion-based encapsulation and delivery systems for polyphenols. Trend Food Sci. Tech. 2016, 47, 1-9.

4. Virtorio, O.; Curcio, M.; Cojoc, M.; Goya, G.F.; Hampel, S.; Lemma, F.; Dubrovska, A.; Crrillo, G. Polyphenols delivery by polymeric materials:challenges in cancer treatment. Drug Delivery 2017, 24, 162-180. 
5. Fang, Z.; Bhandari, B. Encapsulation of polyphenols-A review. Trend Food Sci. Tech. 2010, 21, 510-523.

6. Watson, R.R.; Preedy, V.; S. Zibadi, S. (Eds), Polyphenols in human health and disease. Elsevier 2014.

7. Lu, J.; Zhao, W.; Hang, Y.; Liu, H.; Marquez, R.; Gibbs, R.B.; Li, J.; Venkataramanan, R.; Xu, L.; Li, S. Targeted delivery of doxorubicin by folic acid-decorated dual functional nanocarrier. Mol. Pharm.

2014, 11, 4164-4178.

8. Rollett, A.; Reiter, T.; Nogueir, P.; Cardinale, M.; Loureiroc, A.; Gomes, A.; Cavaco-Paulo, A.; Moreira, A.; Al, A.; Carmo, M.; Guebitz, G.M. Folic acidfunctionalized human serum albumin nanocapsules for targeted drug delivery to chronically activated macrophages. Int. J.Pharm. 2012, 427, 460-466.

9. Yang, C.; Gao, S.; Kjems, J. Folic acid conjugated chitosan for targeted delivery of siRNA to activated macrophages in vitro and in vivo. J. Mater. Chem. B, 2014, 2, 8608-8615.

10. Song, H.; Su, C.; Cui, W.; Zhu, B.; Liu, L.; Chen, Z.; Zhao, L. Folic acidchitosan conjugated nanoparticles for improving tumor-targeted drug delivery. Biomed. Res. Int. 2013, dx.doi.org/10.1155/2013/723158.

11. Stella, B.; Arpicco, S.; Peracchia, M.T.; Desmaële, D.; Hoebeke, J.;. Renoir, M.; D'Angelo, J.; Cattel, L.; Couvreur, P. Design of folic acid-conjugated nanoparticles for drug targeting. J. Pharm. Sci. 2000, 89, 1452-1464.

12. Xu, J.; Xu, B.; Shou, D.; Qin, F.; Xu, Y.; Hu, Y. Characterization and evaluation of a folic acid receptor-targeted cyclodextrin complex as an 
anticancer drug delivery system. Eur. J. Pharm. Sci. 2016, 15, 132-142.

13. Vlahov, I.R.; Leamon, C.P. Engineering folate-drug conjugates to target cancer: from chemistry to clinic. Bioconjugate Chem. 2012, 18, 1357-1369.

14. Wang, Y.; Li, P.; Chen, L.; Gao, W.; Zeng, F.; Kong, L.X. Targeted delivery of 5-fluorouracil to HT-29 cells using high efficient folic acid-conjugated Nanoparticles. Drug Delivery 2015, 22, 191-198.

15. Thornthwaite, J.T.; Shah, H.R.; England, S.R.; Roland, L.H.; Thibado, S.P.; K.Ballard, T.; Goodman, B.S. Anticancer effects of curcumin, artemisinin, genistein and resveratrol, and vitamin C: Free versus liposomal forms. Adv. J. Biol. Chem. 2017, 7, 27-41.

16. Azqueta, A.; Collins, A. Polyphenols and DNA damage: A mixed blessing. Nutrients 2016, 8, 785; doi:10.3390/nu8120785.

17. Chanphai, C.; Konka, V.; Tajmir-Riahi, H.A. Folic acid-chitosan conjugation. A new drug delivery tool. J. Mol. Liquids 2017, 238, 155-159.

18. Liang, L.; Tajmir-Riahi, H. A.; Subirade, M. Interaction of $\beta$-lactoglobulin with resveratrol and its biological implications. Biomacromolecules 2008, 9, $50-56$.

19. Usha, S.; Johnson, I.M.; Malathi, R. Modulation of DNA intercalation by resveratrol and genistein. Mol. Cell. Biochem. 2006, 284, 57-64.

20. Usha, S.; Johnson, I.M.; Malathi, R. Interaction of resveratrol and genistein with nucleic acids. J. Biochem. Mol. Biol. 2005, 38, 198-205.

21. Chignell, C.F.; Bilski, P.; Reszka, K.J.; Motten, A.G.; Sik, R.H.; Dahl, T.A. Spectral and photochemical properties of curcumin. Photochem. Photobiol. 
1994, 59, 295-302.

22. Lakowicz, J.R. In Principles of Fluorescence Spectroscopy, $3^{\text {nd }}$ ed; Springer: 2006, New York.

23. Skovstrip, S.; Hansen, S.G.; Skrydstrup, T.; Schiott, B. Conformational flexibility of chitosan: a molecular modeling study. Biomacromolecules 2010, 11, 3196-3207.

24. Bourassa, P.; Hasni, I.; Tajmir-Riahi, H.A. Folic acid complexes with human and bovine serum albumins. Food Chem. 2011, 129, 1148-1155.

25. Manchanda, R.; Nimesh, S. Controlled size chitosan nanoparticles as anefficient, biocompatible oligonucleotides delivery system. J. Appl. Polymer Sci. 2010, 118, 2071-2077.

26. Hembram, K.C.; Prabha, S.; Chandra, R.; Ahmed, B.; Nimesh, S. Advances in preparation and characterization of chitosan nanoparticles for therapeutics. Artif. Cells Nanomed. Biotechnol. 2016, 44, 305-314.

27. Sathiyabama, M.; Parthasarathy, R. Biological preparation of chitosan nanoparticles and in vitro antifungal efficacy against some phytopathogenic fungi. Carbohydr. Polymers 2016, 151, 321-325.

28. Viveka, R., Nipun Babua, V.; Thangama, R.; Subramanian, K.S.; Kannan, S. $\mathrm{pH}$-responsive drug delivery of chitosan nanoparticles as tamoxifen carriers for effective anti-tumor activity in breast cancer cells. Colloids Surf. B 2013, $111,117-123$.

29. Zhao, Y.; Ma, L. Zeng, R.; Tu, M.; Zhao, J. Preparation, characterization and protein sorption of photo-crosslinked cell membrane-mimicking chitosan- 
based hydrogels. Carbohydr. Polymers 2016, 151, 237-244.

30. Hoven, V.P.; Tangpasuthadol, V.; Angkitpaiboon, Y.; Vallapa, N.;

Kiatkamjornwong, S. Surface-charged chitosan: preparation and protein Adsorption. Carbohydr. Polymers 2007, 68, 44-53.

31. Chanphai, P.; Tajmir-Riahi, H.A. Probing the binding of resveratrol, genistein and curcumin with chitosan nanoparticles. J. Mol. Liquids 2017, 243, 108-114.

32. Chandra, S.; Dietrich, S.; Lang, H.; Bahadur, D. Dendrimer-doxorubicin conjugate for enhanced therapeutic effects for cancer. J. Mater. Chem.

2011, 21, 5729-5737.

33. Brugnerotto, J.; Lizardi, J.; Goycoolea, F.M.; Arguelles-Monal, W.;

Desbrieres, J.; Rinaudo, M. An infrared investigation in relation with chitin and chitosan characterization. Polymer 2001, 42, 3569-3580.

34. Jakobek, L. Interactions of polyphenols with carbohydrates, lipids and Proteins. Food Chem. 2015, 175, 556-567.

35. Hao, J.; Tong, T.; Jin, K.; Zhuang, Q.; Han, T.; Bi, Y.; Wang, J.; Wang, X. Folic acid-functionalized drug delivery platform of resveratrol based on pluronic 127/D- $\alpha$-tocopheryl polyethylene glycol 1000 succinate mixed micelles. Int. J. Nanomed. 2017, 12, 2279-2292. 


\section{Captions for Figures}

Scheme 1. Chemical structures of polyphenols

Figure 1. TEM images showing the morphology of chitosan (A), folic acid-chitosan conjugate (B) with resveratrol (C), genistein (D) curcumin (E) complexes. The concentrations of chitosan and polyphenols were $60 \mu \mathrm{M}$ in all samples.

Figure 2. Fluorescence emission spectra of polyphenols with chitosan-15 kDa for (A) resveratrol in $10 \mathrm{mM}$ Tris- $\mathrm{HCl}$ buffer $\mathrm{pH} 7.2$ at $24{ }^{\circ} \mathrm{C}$ (resveratrol) (a) $20 \mu \mathrm{M}$ and (b-k) chitosan at 2, 5, 10, 15, $2025,30,4050$ and $60 \mu \mathrm{M}$; B (genistein) $(20 \mu \mathrm{M})(\mathrm{b}-\mathrm{k})$ chitosan at 2, 5, 10, 15, $2025,30,4050$ and $60 \mu \mathrm{M}$ and $\mathbf{C}$ (curcumin) $(20 \mu \mathrm{M})(\mathrm{b}-\mathrm{k})$ chitosan at $2,5,10,15,2025,30,4050$ and $60 \mu \mathrm{M}$; Inset The plot of $F_{0} /\left(F_{0}-F\right)$ as a function of 1/acid-chitosan concentration. The binding constant $K$ being the ratio of the intercept and the slope for polyphenol-acid-chitosan conjugates.

Figure 3. Fluorescence emission spectra of polyphenosl with chitosan-100 kDa for (A) resveratrol in $10 \mathrm{mM}$ Tris- $\mathrm{HCl}$ buffer $\mathrm{pH} 7.2$ at $24{ }^{\circ} \mathrm{C}$ (resveratrol) (a) $20 \mu \mathrm{M}$ and (b-k) chitosan at 2, 5, 10, 15, $2025,30,4050$ and $60 \mu \mathrm{M}$; B (genistein) $(20 \mu \mathrm{M})(\mathrm{b}-\mathrm{k})$ chitosan at 2, 5, 10, 15, $2025,30,4050$ and $60 \mu \mathrm{M}$ and $\mathbf{C}$ (curcumin) $(20 \mu \mathrm{M})(\mathrm{b}-\mathrm{k})$ chitosan at 2, 5, 10, 15, $2025,30,4050$ and $60 \mu \mathrm{M}$. Inset The plot of $F_{0} /\left(F_{0^{-}} F\right)$ as a function of 1/acid-chitosan concentration. The binding constant $K$ being the ratio of the intercept and the slope for polyphenol-acid-chitosan conjugates.

Figure 4. FTIR spectra in the region of $1800-600 \mathrm{~cm}^{-1}$ of hydrated films (pH 7.2) for free folic acid-chitosan-15 kDa for (A) resveratrol, (B) genistein and (C) curcumin), chitosan $(60 \mu \mathrm{M})$ with difference spectra (diff.) of polyphenol-acid-chitosan conjugates 
(bottom two curves) obtained at different polyphenol concentrations (indicated on the figure).

Figure 5. FTIR spectra in the region of $1800-600 \mathrm{~cm}^{-1}$ of hydrated films (pH 7.2) for free folic acid chitosan-100 kDa for (A) resveratrol, (B) genistein and (C) curcumin), chitosan $(60 \mu \mathrm{M})$ with difference spectra (diff.) of polyphenol-acid-chitosan conjugates (bottom two curves) obtained at different polyphenol concentrations (indicated on the figure).

Figure 6. Best conformations for polyphenols docked to folic acid (A) and chitosan (B) with the free binding energy for resveratrol, genistein and curcumin (polyphenols is shown in green color). 
Table 1. Calculated binding constants $(K)$ for the polyphenol-folic acid chitosan nanocapsules with the number of bound polyphenol $(n)$ per nanocarrier and loading efficacy (LE)

\begin{tabular}{|c|c|c|c|}
\hline Complexes & $\begin{array}{c}K(\mathrm{~mol} / \mathrm{L})^{-1} \\
\left(10^{6}\right)\end{array}$ & $n$ & LE \% \\
\hline Resveratrol-folic acid-chitosan-15 & $4.4( \pm 1)$ & 1.5 & 60 \\
\hline Genistein-folic acid-chitosan-15 & $0.74( \pm 0.05)$ & 1 & 50 \\
\hline Curcumin-folic acid-chitosan-15 & $0.76( \pm 0.08)$ & 1 & 50 \\
\hline Resveratrol-folic acid-chitosan-100 & $5.9( \pm 0.9)$ & 1.5 & 65 \\
\hline Genistein-folic acid-chitosan-100 & $1.5( \pm 0.01)$ & 1.1 & 55 \\
\hline Curcumin-folic acid-chitosan-100 & $1.7( \pm 0.2)$ & 1.2 & 55 \\
\hline
\end{tabular}


<smiles>Cc1cc(O)cc(/C=C/c2ccc(O)cc2)c1</smiles>

OH<smiles>O=C1C(c2ccc(O)cc2)=COC2C=C(O)C=C(O)C12</smiles>

Genistein<smiles>COc1cc(/C=C/C(=O)CC(=O)/C=C/c2ccc(O)c(OC)c2)ccc1O</smiles>

Curcumin

Scheme 1: Chemical structures of polyphenols 


\section{Figure 1}
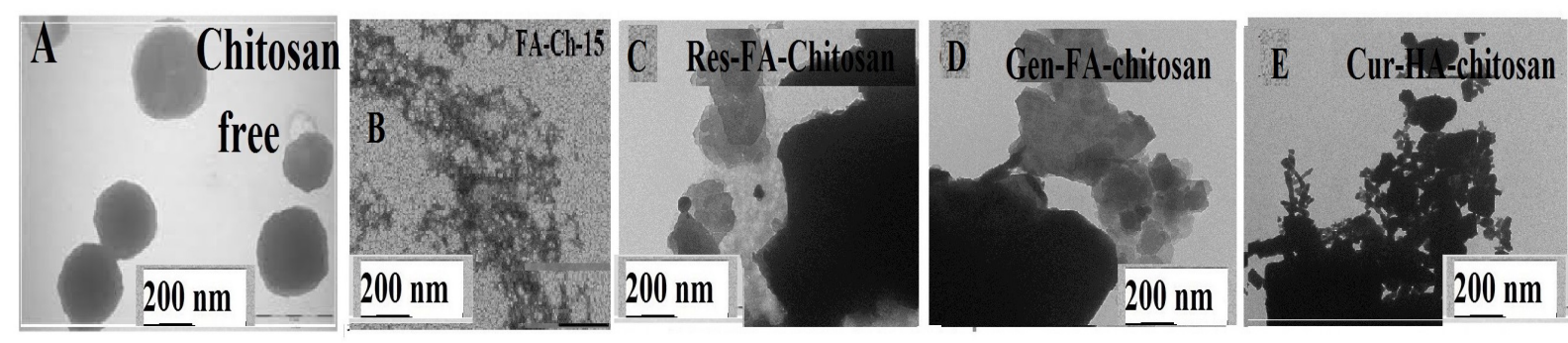
Figure 2

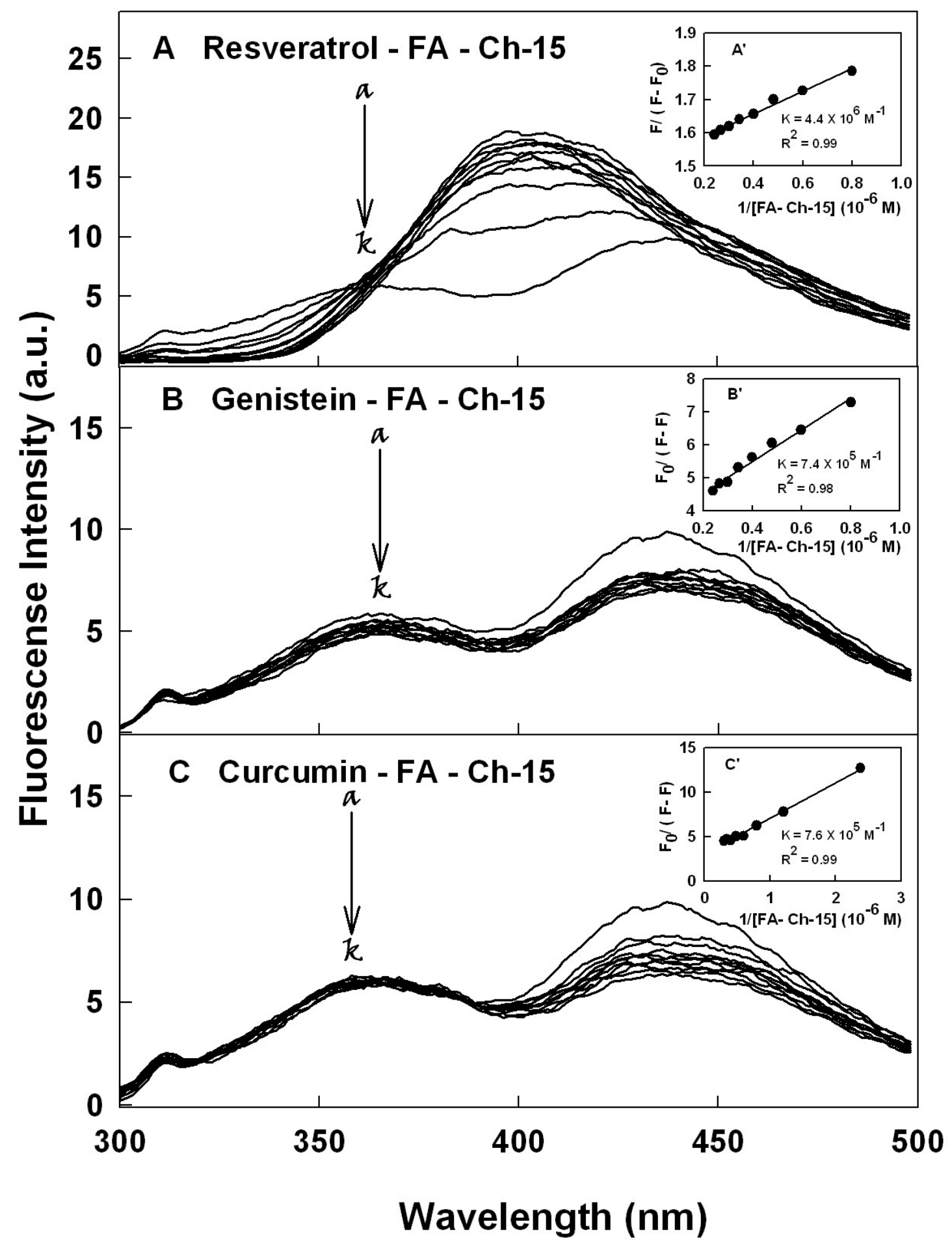


Figure 3

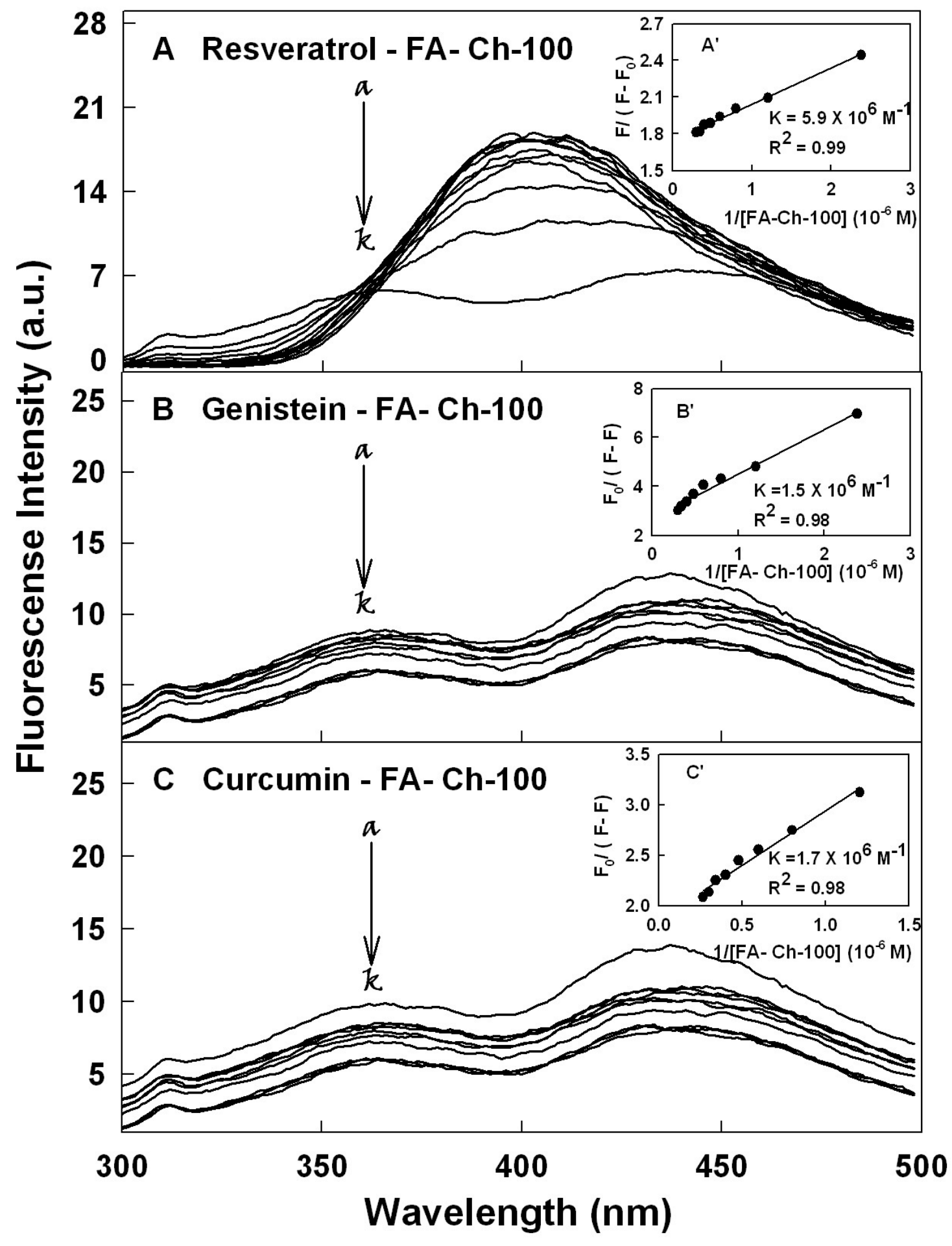


Figure 4

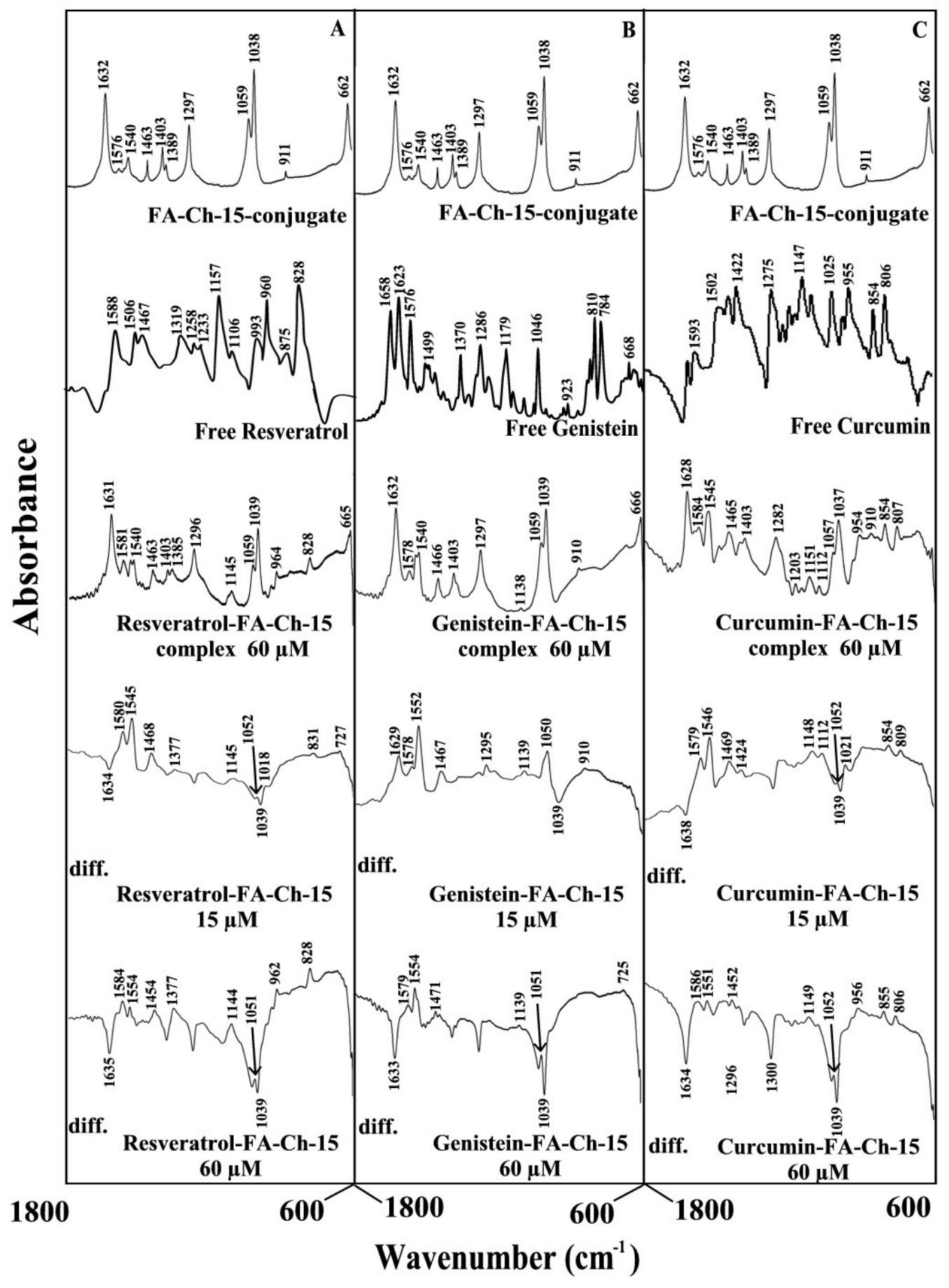


Figure 5

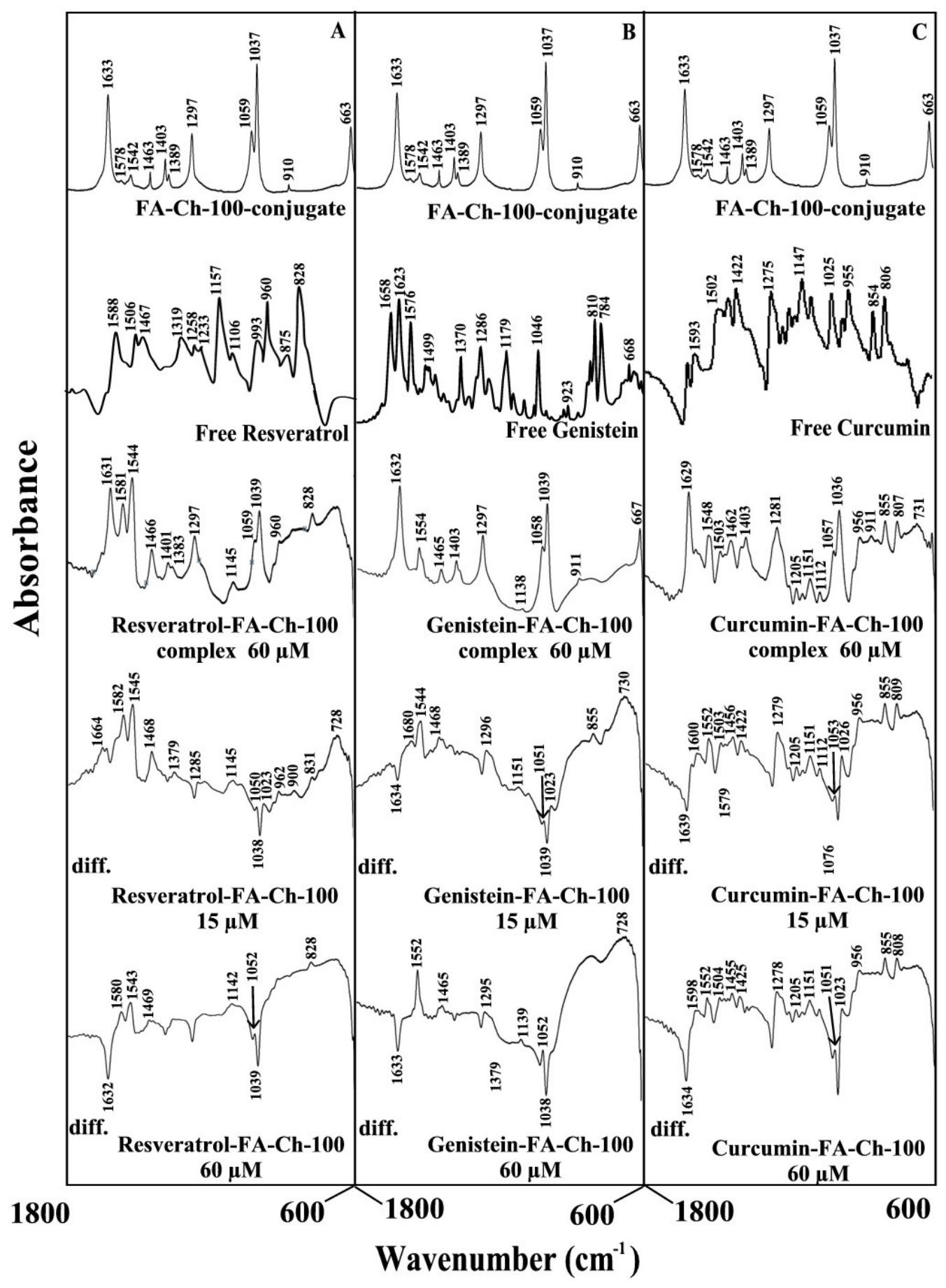


Figure 6

Resveratrol - Folic acid

